\title{
The relative difficulty of a subject-generated rule in an attribute identification task
}

\author{
RICHARD L. GOTTWALD* and MICHAEL R. SWAINE $\dagger$ \\ Indiana Lniversity' at South Bend, South Bend, Ind. 46615
}

Comparisons of attribute identification difficulty were made among a S-generated conceptual rule (double conjunction) and two commonly studied conceptual rules (biconditional and exlusive disjunction). The three rules described identical subsets of stimuli. The double conjunction was the easiest. The other two rules did not differ in difficulty. There were also effects due to practice and the salience of the relevant attributes, but there were no significant interactions among the three variables (rule, practice, attribute salience).

Concept attainment may be viewed as a learning task in which $\mathrm{S}$ must learn to identify some subset of stimuli. Usually $S$ is expected to formulate a rule involving one or more relevant attributes. Haygood \& Bourne (1965) have demonstrated the feasibility of analyzing this task into two components: attribute identification, a procedure in which $S$ is told the rule but the relevant attributes are unknown; and mule learning, a procedure in which $S$ is told the critical values of the relevant at tributes but the rule is unknown.

Usually several different conceptual rules may be used to describe a subset of stimuli. For example, consider the subset (red triangle, green square) of the set of stimuli generated by the two binary attributes of color (red and green) and form (triangle or square). This subset may be described by an exclusive disjunction rule (exemplars of the concept are red or square but not both red and square), by a biconditional rule (red and triangle or not-red and not-triangle), or by a rule we shall term a double conjunction (red and triangle, green and square).

In most attribute identification studies (e.g., Haygood \& Bourne, 1965), the critical levels of the relevant attributes are held constant, but the composition of the positive and negative subsets varies across concepts. In the above examples, the relevant attributes are held constant and the composition of the positive and negative subsets remains the same but the critical values of the relevant attributes vary across concepts. It has not yet been determined whether the type of rule will affect the difficulty of attribute identification when the composition of the positive and negative subsets is held constant. One purpose of the present study was to answer this question.

Typically, concept at tainment studies have used rules based upon a variety of logical connectives between attribute levels, e.g., conjunction, inclusive or exclusive disjunction, conditional, biconditional, etc. However,

\footnotetext{
* Requests for reprints should be sent to Richard L. Gottwald Department of Psychology, Indiana University at South Bend, South Bend, Ind. 46615.

+ Now at Indiana University at Bloomington, Ind. 47401.
}

Gottwald (1971a,b) examined Ss' protocols in a complete learning task (attributes and rule both unknown), and found that almost all Ss used rules based upon simple affirmations and conjunctions, although other kinds of rules would have been equally correct. Thus, in the example given above, most Ss would be expected to use the double conjunction rule. Indeed, Gottwald (1971a, b) found that 23 out of 23 Ss did use the double conjunction rule in logically similar concepts.

One would expect attribute identification to be easier if the rule was one frequently verbalized by Ss rather than a rule that was rarely verbalized, even though it might be equally correct. However, the fact that Ss use a certain rule does not necessarily mean that that rule is easier than other possible rules. The present study was designed to evaluate the relative difficulties of the double conjunction, biconditional, and exclusive disjunction rules in attribute identification.

\section{METHOD \\ Subjects}

A total of 48 Ss ( 26 male and 22 female) was drawn from the introductory psychology pool at Indiana University at South Bend. Course credit was given for participation in the experiment.

\section{Stimuli and Concepts}

The stimuli were schematic drawings of "bugs" which varied on four binary attributes: body shape, spot color, leg number, and antenna type. The relevant attributes were either body shape and spot color or body shape and antenna type. Preliminary work with a free classification task showed that the pair of body shape and spot color was high in salience (used in $40.4 \%$ of the classifications) and the pair of body shape and antenna type was moderately low in salience (used in $11.9 \%$ of the classifications). The concepts were logically similar to the above example and could be described by either a double conjunction, biconditional, or exclusive disjunction rule.

\section{Procedure}

The $\mathrm{S}$ was told that he was in a learning experiment and that his task was to learn to identify a class of bug which was labeled alpha. The $S$ was shown both values of each of the four attributes and he was given one of the three rules as the rule that would identify the alphas. The rule was carefully described to S, and the rule with an example was on a card available to $S$ throughout the experiment.

Each $\mathrm{S}$ was tested on two attribute identification problems involving only one rule. For one-half of the Ss, body and spots were relevant in the first problem and body and antenna were relevant in the second. For the other half of the Ss, this order was reversed. A set of 16 stimuli was prepared for each problem. The stimuli were presented to each $\mathrm{S}$ in four random orders. The stimuli were in the form of slides, and were back-projected for $8 \mathrm{sec}$ with $1 \mathrm{sec}$ between slides.

When a stimulus appeared, $\mathrm{S}$ responded verbally and $\mathrm{E}$ gave immediate verbal feedback. After $\mathrm{S}$ had responded to one order of the set of 16 stimuli, he was asked to write a complete rule which would identify the alphas. Guessing was encouraged. Each 
Table 1

Mean Trials to Last Error

\begin{tabular}{lccc}
\hline & \multicolumn{3}{c}{ Order of Learning } \\
\cline { 2 - 4 } \multicolumn{1}{c}{ Conceptual Rule } & First & Second & Mean \\
\hline Double Conjunction & 58.2 & 31.2 & 44.7 \\
Biconditional & 85.1 & 55.6 & 70.4 \\
Exclusive Disjunction & 79.9 & 74.1 & 77.0 \\
Mean & 74.4 & 53.6 & \\
\hline
\end{tabular}

rule was examined and, if it was not the correct type of rule, E explained the rule again. This procedure was repeated until $S$ reached a criterion of one perfect classification of the set of 16 stimuli or until $\mathrm{S}$ had responded to a total of 160 stimuli.

\section{RESULTS AND DISCUSSION}

Table 1 shows mean trials to criterion (excluding the 16 criterion trials) for the various conditions. The three conceptual rules differed in difficulty, $F(2,42)=3.80$, $\mathrm{p}<.05$. The double conjunction was easier than the other two rules, smaller $F(1,42)=4.98, p<.05$, and the exclusive disjunction and biconditional were not significantly different $(F<1.0)$. The task was easier when the relevant attributes were high in salience, $\mathrm{F}(1,42)=4.18, \mathrm{p}<.05$. Mean trials to criterion was 54.4 when body shape and spot color were relevant and 73.7 when body shape and antenna type were relevant. There was also a significant practice effect, $F(1,42)=$ $4.83, \mathrm{p}<.05$. Mean trials to criterion for Problem 1 was 74.4 and for Problem 2, 53.6. None of the interactions was significant (smallest $\mathrm{p}>.10$ ).

These results show that the difficulty of attribute identification is affected by the type of rule even when the composition of the stimulus subsets is held constant. In addition, the S-generated rule, double conjunction was easier than the two commonly studied rules, biconditional and exclusive disjunction. Informal observation suggests that $\mathrm{Ss}$ had less trouble understanding the double conjunction than the other two rules when they were first informed about the nature of the rules. However, all Ss were using the correct type of rule by the end of the first problem, and the differences in difficulty were still present in the second problem. Thus, the pattern of results is due to more than initial difficulty in understanding and probably reflects some inherent differences among the rules. It is not surprising that the biconditional and exclusive disjunction did not differ in difficulty, because previous studies also found no difference in difficulty between the two rules, both in a complete learning task (Neisser \& Weene, 1962) and in an attribute identification task using the more conventional methodology (Giambra, 1971; Laughlin, 1968).

Gottwald (1971c) found that the results of a free classification task could be used to predict the salience of attribute pairs in a complete learning task using a double conjunction type of concept. The present results confirm this finding and extend it to the attribute identification task.

\section{REFERENCES}

Giambra, L. M. Selection strategies for eight concept rules with non-exemplar start cards. Journal of Experimental Psychology, 1971, 87, 78-82.

Gottwald, R. L. Attribute-response correlations in concept attainment. American Journal of Psychology, 1971a, 84, 425-436.

Gottwald, R. L. Effects of response labels in concept attainment. Journal of Experimental Psychology, 1971b, 91, 30-33.

Gottwald, R. L. Attribute salience and attribute-response correlations in concept attainment. Paper presented at the meeting of the Eastern Psychological Association. New York, April 1971c.

Haygood, R. C., \& Bourne, L. E., Jr. Attribute- and rule-learning aspects of conceptual behavior. Psychological Review, 1965 , 72, 175-195.

Laughlin, P. R. Focusing strategy for eight conceptual rules. Journal of Experimental Psychology, 1968, 77, 661-669.

Neisser, U., \& Weene, P. Hierarchies in concept attainment. Journal of Experimental Psychology, 1962, 64, 640-645.

(Received for publication September 18, 1973.) 